\title{
DISCUSSION
}

\section{ZEILICOVICI ON TEMPORAL BECOMING}

\section{NATHAN OAKLANDER}

The aim of David Zeilicovici's recent article "Temporal becoming Minus the Moving Now" is clear and admirable. ${ }^{1}$ He wants to develop a theory of temporal becoming that (1) gives full ontological status to B-relations, (2) gives full ontological status to the transitory aspect of time and (3) avoids commitment to the moving-NOW and the subsequent (McTaggart's) paradox. It does not seem to me that he succeeds in accomplishing these difficult undertakings and in what follows I shall attempt to explain why.

A useful place to begin is by briefly considering the theory that he wants to avoid. On the traditional tensed, or A-theory of time, the NOW is a particular or a property that moves along an ordered, but as yet non-temporal, C-series. The terms of the C-series exist (tenselessly) in unchanging relations to each other, and these unchanging relations become temporal relatons as the NOW moves across them so that one term, e is NOW when another term, $e^{\prime}$ is future and then, when the NOW "hits" $\mathrm{e}$ ', that is, when $e^{\prime}$ is present, $\mathrm{e}$ is past. The problem is that this account of time presupposes time. As the words "and then" and "when" indicate, in order for there to be temporal relations among terms in the $\mathrm{C}$-series, there have to be temporal relations among the "events" of $e$ 's being NOW and $e$ 's not being NOW, otherwise we have a contradiction. But given the tradiional view, in order for there to be temporal relations between terms there must be a NOW moving across them. Thus, we are either caught in a vicious circle (of trying to define temporal relations in terms of the moving NOW and the moving NOW in terms of term relations) or a vicious infinite regress (where the contradiction of every 


\section{NATHANOAKLANDER}

term being past, present, and future, or NOW and not NOW, is passed on from one series of terms to another).

At this point, Zeilicovici offers a theory that attempts to avoid the pitfalls of the traditional view by maintaining both that (i) at any present moment future time does not exist and (ii) the B-series is a single time series. On this view, temporal becoming involves a change of time, and such a change involves the creation of a

new entity [an instantaneous A-series] which did not exist (not even as an incomplete series) before that which makes the new A-determination true (i.e. the addition) is there. (p. 518)

Thus, change of time consists in the "replacement" of an old Aseries by a new A-series. What is crucial to note about "replacement" is that "the increase in the sum total of existence" is not an increase is a previously existing A-series, for if it was then we would have a change in time and not a change of time. Rather, as moments are created a totally new A-series comes into existence and the old A-series ceases to exist, i.e., is replaced.

Zeilicovici's view is intriguing, but we must clarify certain points in it before we can detect its strenghts or weaknesses. For example, what does he mean by "moments" of the "B-series" and the "A-series"? Zeilicovici's official view of "moments" is relational. He says that "time is the ordered set of moments, and moments are defined as equivalence sets of events under the relation of simultaneity." ( $p$. 509) and his response to Melvin Schuster's argument against the creationist view of time seems to imply a commitment to the relational theory. For on the event-creationist view events come into being at "at already existing moments" (and so is vulnerable to the moving NOW critique), but on the moment-creationist view that Zeilicovici adopts "future moments are not a-priori existent delivery rooms in which events are to be born...." (pp. 510-511) (and so, allegedly, is not open to Schuster's criticism). Thus, on Zeilicovici's official view, there do not exist (tenselessly) absolute moments that events come into existence (or are created) at, but rather, what comes into existence are moments relationally understood, i.e., sets of simultaneous events. ${ }^{2}$

Nevertheless, other rmearks Zeilicovici makes wo suggest a commitment to absolute time. He claims that A-theorists no less than 
B-theorists can maintain that the B-series (by itself) is a time series. After all, he exclaims,

Why are we not free to grant that earlier than is "an analyzable temporal relation" ... just because it ranges over the unanalyzable temporal particulars known as moments? (pp. 513-514)

Soon we shall see why an A-theorist is not free to grant this, but presently we should note that no relationalist would countenance "unanalyzable temporal particulars know as moments." One might say that Zeilicovici just misspoke here, but then what are we to make of his claim that

if $\mathbf{t}<\mathrm{t}^{\prime}, \mathrm{t}$-tokens of such statements as " $\mathrm{t}$ ' exists at $\mathrm{t}^{\prime \prime}$ are not always as uninformative as they look. For, if $t$ happens to be the present, $t^{\prime}$ may remain forever just an empty placeholder on the time axis, while the above ttoken conveys the informative prediction that this is not the case. (p. 509; emphasis added)

What can he mean by saying that " $\mathrm{t}$ ' can remain an empty placeholder on the time axis" if not that $t^{\prime}$ may exist as a term in the B-series even if no event (or substance) comes to occupy it? And that is to be committed to absolute time. The ambiguity reflected in his talk about "moments" has an analogue in his account of the B-series.

The B-series is, according to Zeilicovici, a genuinely temporal series whose generating relation is being later than. Furthermore, the B-series is "static" or unchanging in that neither the relations between the terms in the series, nor the series as whole changes any of its properties. Most importantly, there is one and only one B-series. Zeilicovici's reasoning for that thesis is important and worth guoting at length.

The single B-series differs from any of the many Aseries just in failing to distinguish between existing moments and predicted moments and by being, as a consequence, unbounded. B-sentences report B-relations between existing temporal particulars and predict them for non-existing ones without registering any 


\section{NATHANOAKLANDER}

ontological difference between the two kinds of particulars or any epistemological difference between the two kinds of acts. ... (p. 511)

The addition of a new moment to existing time fails to create a new B-series because the new moment appears in the old B-series anyway. Thus the two series are indistinguishable from each other and so must, a fortiori,e counted as one and the same series. It is, of course, true that what appears in the new series is a "real", existing moment, while what figures in the old series is a mere predicted shadow of a moment. But such a feature cannot be used as a springboard for making a distinction between two B-series because Being later than, the generating relation of any B-series, signally fails to mark any such distinction between the two kinds of moments. (p. 512)

His position seems to be this: all the terms that are ever in the Bseries are always in the B-series. There is a single B-series composed of the same terms at every (present) moment, and that is so even though at every present moment some terms of the B-series exist and some terms of the B-series (those that are predicted) do not exist, i.e., they are terms beyond the upper bound. But what does it mean to say that an existing moment in the "new" B-series "figures in the old series \{as\} a mere predicted shadow of a moment"? (p. 512; my emphasis)

Either the predicted shadow is a member of the B-series or it is not. If it is not a member of the B-series, then the new B-series is not the same as the old B-series. On the other hand, if the predicted shadow is a member of the B-series, then it would appear that it is nothing other than a "placeholder" on the (absoulte) time axis "waiting" to be filled by newly created events. In the first case, he must abandon his claim to be treating the B-series (by itself) as a temporal series independently of the phenomenon of temporal becoming as represented by the A-series. In the second case, he must abandon his claim to be analyzing temporal becoming minus the moving-NOW.

To connect this point with the previous one about the ambiguity concerning "moments" note that if moments are understood relationally then the "predicted shadow" cannot exist as part of a single B-series. For if the predicted moments are sets of simultaneous events, then at 
any present moment there are an infinite or at least an indefinite number of (future) sets of simultaneous events. After all, on this view the future is open. But to say that the future is open is just to say that there are many possible events that will follow the present moment. Each possibility constitutes a different B-series. Admittedly, there is only one actual B-series, but the actual B-series, the single B-series, is composed entirely of terms that exist. Thus, if he adopts a relational view of moments, and if he maintains that the future does not exist, but is only predicted or conjectured, then he cannot speak of a singly Bseries, that is, he cannot say that the old B-series is the same as the "new" B-series. Indeed, he cannot say that the B-series is a time series independently of temporal becoming at all because it turns out that the $\mathrm{B}$-series simply is the A-series. If, however, moments are understood as absolute, that is, as intrinsically temporal particulars, then predicted moments are part of a single B-series and the becoming of events, or the transitory aspect of time, must reintroduce the moving-NOW. By playing on the ambiguity of "moments" Zeilicovici is able to think a change of time preserves a single B-series (where moments are absolute) within which there exists the creation of different (new) Aseries (where moments are construed relationally.) Perhaps we can understand this last point more clearly by considering his conception of the A-series.

Simply put, the A-series is that part of the B-series that exists. As he puts it:

A present-at-t event \{is\} any event occurring at the upper bound of until-t existing time. If some predictions are fulfilled, there will be a next moment; $t$ will lose the frontier characteristic and our event will lose presentness while retaining intact all its ordinary properties and all its temporal order B-relations to its previous partners. But, because it is now related to new members, this event (and all others) will belong to a different timeseries, the series which includes the previous one but whose "membership is increased." (p. 511)

Thus, the passage of time is the creation of new A-series, each containing more members than the previous one that it has replaced. Thus, he thinks that he has a single B-series, and within that single Bseries a multitude of A-series. In this way there exists a change of time 


\section{NATHANOAKLANDER}

(A-series) without a change in time, that is, without any single thing (like the NOW) moving along the B- (or C-) series. But given Zeilicovici's problems with the B-series his attempt to distinguish a change of time from a change in time fails.

If the B-series is composed of absolute moments "waiting" to be occupied, then the creation of each new A-series at a certain time is itself an event that must take place in time and so must undergo temporal becoming. On the other hand, if the B-series is composed of moments construed as sets of simultaneous events, and future moments do not exist as members of the B-series, then we cannot speak of a single B-series, or an "old" B-series being the same as the "new" B-series. Rather, as each moment is created we get a different Aseries which itself is a B-series, and that is precisely the part of the traditional A-theory that Zeilicovici so strenuously wants to avoid. Finally, if he maintains the relational view of moments and insists that there is only one B-series (which I have argued all of whose terms must exist), then obviously Broad's idea that "The sum total of existence is always increasing" must be abandoned. Indeed, it would appear that the only way to reintroduce transiency at this point is by appealing to the moving-NOW. On the hopelessness of that way of trying to make sense of temporal becoming both Zeilicovici and I agree.

\section{THE UNIVERSITY OF MICHIGAN-FLINT} FLINT, MICHIGAN 48502

USA

\section{NOTES}

1 David Zeilicovici, "Temporal Becoming Minus the Moving-Now," NOUS, 23 (1989), pp. 505-524. All page references in the text will be to this paper.

2 Note that Zeilicovici does emphasize that each A-series is instantaneous, but this is troublesome because each A-series, in order to have its membership increased, must contain past as well as present members. However, if a newly created A-series contains members that are both past and present, then how can what is created be a moment, i.e., a set of simultaneous events? 